\title{
Singular values and bounded Siegel disks
}

\author{
BY ANNA MIRIAM BENINI \\ Dip. di Matematica, Universita' di Tor Vergata \\ Via della Ricerca Scientifica \\ 00133 Roma, Italy \\ AND NÚRIA FAGELLA \\ Dept. de Matemàtiques i Informàtica \\ Barcelona Graduate School of Mathematics (BGSMath) \\ Gran Via 585, 08007 Barcelona, Spain
}

(Received 2 June 1994; revised 3 April 1995)

\begin{abstract}
Let $f$ be an entire transcendental function of finite order and $\Delta$ be a forward invariant bounded Siegel disk for $f$ with rotation number in Herman's class $\mathcal{H}$. We show that if $f$ has two singular values with bounded orbit, then the boundary of $\Delta$ contains a critical point. We also give a criterion under which the critical point in question is recurrent. We actually prove a more general theorem with less restrictive hypotheses, from which these results follow.
\end{abstract}

\section{Introduction}

We consider the dynamical system generated by the iterates of an entire transcendental function $f: \mathbb{C} \rightarrow \mathbb{C}$, that is a function which is holomorphic on the complex plane $\mathbb{C}$ and has an essential singularity at infinity (in general, we will omit the word 'transcendental'). In this setup, there is a dynamically natural partition of the phase space into two completely invariant subsets: the Fatou set $\mathcal{F}(f)$, formed by those $z \in \mathbb{C}$ for which the family of iterates $\left\{f^{n}\right\}_{n \in \mathbb{N}}$ is normal in the sense of Montel in some neighborhood of $z$; and the Julia set $\mathcal{J}(f)$, its complement. Orbits in the Julia set exhibit chaotic behavior - in fact, $\mathcal{J}(f)$ is the closure of the repelling periodic points of $f$.

The Fatou set is open, possibly with infinitely many components, called Fatou components. The periodic ones are completely classified into basins of attraction of attracting or parabolic cycles, Siegel disks (topological disks on which a certain iterate of $f$ is conjugate to a rigid irrational rotation of angle $\theta$, called the rotation number) or Baker domains (regions on which iterates converge uniformly to infinity). Non-periodic Fatou components are called preperiodic if they are eventually mapped to a periodic component, and wandering otherwise. Baker and wandering domains are types of Fatou components which appear only in the transcendental setting. For a classification of Fatou components in the transcendental case see e.g. [Be].

In holomorphic dynamics, the singular values of $f$ play a crucial role. A singular value of $f$ is a point around which not all branches of $f^{-1}$ are well defined and univalent (see Section $2 \cdot 1$ ). With this 
definition, the set

$$
S(f):=\{s \in \mathbb{C} ; s \text { is a singular value for } f\} .
$$

is closed. Observe that $f: \mathbb{C} \backslash f^{-1}(S(f)) \rightarrow \mathbb{C} \backslash S(f)$ is a covering of infinite degree. A singular value $s \in \mathbb{C}$ is called a critical value if $s=f(c)$, where $c$ is a point with vanishing derivative or critical point. It is called an asymptotic value if there exists a curve tending to infinity whose image under $f$ is a bounded curve converging to $s$. The set of singular values consists of critical values, asymptotic values and any of their accumulation points. Singular values may also have regular preimages, i.e. preimages at which $f$ is locally univalent. A singular value $s$ is called isolated if there is a neighborhood $V$ of $s$ such that $V \cap S(f)=\{s\}$. Let $s$ be a singular value or a critical point. The $\omega$-limit set of $s$ is defined as the set of accumulation points of the orbit of $s$, and is denoted by $\omega(s)$. The point $s$ is called recurrent if and only if it belongs to its $\omega$-limit set.

Most types of Fatou components are associated with a singular orbit (the orbit of a singular value). For example, all basins of attraction of attracting or parabolic cycles must contain at least one singular orbit [Mi, Theorems 8.6 and 10.15] which therefore accumulates on the periodic cycle.

Siegel disks cannot contain critical points although they may contain asymptotic or critical values if these have regular preimages which are contained in the cycle of Siegel disks. On the other hand, an important interplay between singular orbits and boundaries of Siegel disks is highlighted by a theorem of Fatou, which we state in the version of [Mi, Corollary 14.4].

Fatou's Theorem. If $z_{0}$ is a point on the boundary of a Siegel disk, then every neighborhood of $z_{0}$ contains infinitely many points belonging to the postsingular set.

The relation of $S(f)$ with wandering or Baker domains is not as well understood but, if $f$ is an entire function with finitely many singular values, neither wandering domains nor Baker domains exist ([EL, GK $]$ ). Likewise, if $f$ belongs to the Eremenko-Lyubich class

$$
\mathcal{B}:=\{f: \mathbb{C} \rightarrow \mathbb{C} \text { entire transcendental; } S(f) \text { is bounded }\},
$$

then $f$ has no Baker domains, nor any wandering domains for which the iterates converge uniformly to infinity $[\mathbf{E L}]$. Wandering domains whose orbit accumulates both in a compact set and at infinity for functions in class $\mathcal{B}$ are possible $[\mathbf{B i}$ ], and wandering domains with bounded orbits (that is, whose limit functions do not include infinity) have not yet been discarded, nor constructed.

From now on, let $\Delta$ be a bounded invariant Siegel disk with rotation number $\theta$. Although the topology of $\Delta$ is trivial and the dynamics is well understood, neither assertion is true for the boundary of the Siegel disk. Certain properties depend on the rotation number $\theta$, and in some instances, on the dynamics of $f$ outside of $\Delta$. For example, it is not known (even in rational or polynomial dynamics) whether $\partial \Delta$ is always a Jordan curve, although it is believed to be so. In transcendental dynamics, unbounded Siegel disks may have non-locally connected boundaries due to the presence of an asymptotic value in the boundary (using its preimage at infinity), as occurs for example for some members of the exponential family $\lambda e^{z}$. In the case of bounded disks however, no example has been found yet for which the boundary is not a Jordan curve.

The arithmetics of $\theta$ play a relevant role. The set of Herman numbers $\mathcal{H}$ is defined as the set of $\theta \in \mathbb{R}$ such that every orientation preserving analytic diffeomorphism of the circle is analytically linearizable, that is, analytically conjugate to the rigid rotation $\mathcal{R}_{\theta}$. This set was proven to be nonempty by Herman $[$ He1] who showed that it contains all diophantine numbers, so in particular $\mathcal{H}$ has full measure. The set $\mathcal{H}$ was later characterized arithmetically by Yoccoz $[\mathbf{Y o}]$ in terms of the coefficients of the continued fraction expansion. The set $\mathcal{H}$ is larger than the set of numbers of bounded type, that is, the numbers for which the entries of the continued fraction expansion are bounded. By a result of Graczyk and 
Światek [GS] there is always a critical point on the boundary of Siegel Disks of holomorphic maps with bounded-type rotation number, provided the Siegel disk is fully contained in the domain of definition of the map. In our case, the assumption of being fully contained in the domain of definition of the map is equivalent to the Siegel disk being bounded.

The existence of an asymptotic value (acting as such) or a critical point in the boundary of a Siegel disk is an obvious obstruction for the linearization domain to be extended further and hence a natural reason for the maximality of $\Delta$. A theorem of Ghys $[\mathbf{G h}$, Theorem 3] states that if $f$ has rotation number $\theta \in \mathcal{H}$ on an invariant Jordan curve $\gamma$ and $f$ is univalent in a neighborhood of $\gamma$, then $f$ is analytically conjugate to the rigid rotation $R_{\theta}$ in an annular neighborhood of $\gamma$. The main corollary is that if $\theta \in \mathcal{H}$ and $\partial \Delta$ is a Jordan curve then, independently of the nature of $f$ outside the disk, $\partial \Delta$ contains a critical point. The proof of this theorem is at the base of many later results related to the problem of existence of critical points on the boundary of Siegel disks, which is the main problem addressed in this paper. Finally observe that that the arithmetic assumption on $\theta$ is non removable, as shown by the examples of Siegel disks with Jordan boundaries without singular values constructed by Ghys [Gh, Theorem 5], Herman [He3] and Rempe [Re1, Theorem 6.1.4] for exponential maps.

The question remains of whether any Siegel disk $\Delta$ with rotation number $\theta \in \mathcal{H}$ contains a critical point (or an asymptotic value) on its boundary, without any assumption on the topology of $\partial \Delta$.

This question was first addressed by Herman [He2] who, based on Ghys' ideas, developed a general theorem about extensions of holomorphic maps which leave invariant a rotation annulus with rotation number in $\mathcal{H}$. His result and some interesting corollaries are summarized in the following statement. Theorem (Herman).

(a) Let $f$ be a holomorphic map on a domain $U \subset \mathbb{C}$, and $\Delta$ be a bounded invariant Siegel disk of $f$ with rotation number $\theta \in \mathcal{H}$ such that $\bar{\Delta} \subset U$. Then if $\left.f\right|_{\partial \Delta}$ is injective there is a critical point in $\partial \Delta$.

(b) If $f(z)=z^{d}+c$ with $d \geq 2$ and $c \in \mathbb{C}$ has a Siegel disk $\Delta$ with rotation number $\theta \in \mathcal{H}$, then $\partial \Delta$ contains a critical point.

(c) If $f(z)=\lambda e^{z}$ with $\lambda \in \mathbb{C} \backslash\{0\}$ has a Siegel disk $\Delta$ with rotation number $\theta \in \mathcal{H}$, then $\Delta$ is unbounded.

Hence part (b) gives an answer for unicritical polynomials. Very recently Chéritat and Roesch [CR, Theorem 1] went one step further and proved that Siegel disks of polynomials with two critical values and rotation number in $\mathcal{H}$ have a critical point on the boundary. They conjecture that this will be the case for all rational maps.

Theorem (Chéritat-Roesch). Let $P$ be a polynomial with two finite critical values, and $\Delta$ be an invariant Siegel disk with rotation number in $\mathcal{H}$. Then $\partial \Delta$ contains a critical point.

Part (c) of Herman's theorem deals with the exponential family, which has one asymptotic value and no critical points. Rempe [Re2] showed additionally that such unbounded Siegel disks must contain the asymptotic value on the boundary. He later considered a more general setting in [Re4] where he showed that if an entire transcendental map has a Siegel disk $\Delta$ with rotation number in $\mathcal{H}$, then the boundary of $\Delta$ contains a singular value, provided that $S(f) \subset J(f)$.

Inspired by the Theorem by Chéritat and Roesch, our goal is to address the problem for entire transcendental functions having a bounded invariant Siegel disk $\Delta$. Intuitively, if the Siegel Disk is bounded and the rotation number is sufficiently well behaved, the Siegel disk interacts with the critical values as in the rational case, and not with the asymptotic values as in a typical transcendental case. Nevertheless, although in some cases we can indeed reduce locally to the polynomial setting, in general we must take into account the very different nature of $f$ provided by its essential singularity at infinity. 
The infinite degree and the presence of asymptotic values give rise to more possibilities than those encountered in the polynomial setting.

Our main result (in its weakest and simplified form) is the following:

Main Theorem (Simplified form). Let $f$ be an entire function of finite order with at most two singular values and with bounded singular orbits. Suppose $f$ has a (bounded) invariant Siegel disk $\Delta$ with rotation number in $\mathcal{H}$. Then there is a critical point on $\partial \Delta$.

We observe that, by the Theorem of Fatou above, the fact that the singular orbits are bounded implies that any Siegel disk for $f$ has to be bounded.

We do not prove this theorem directly. Instead, we state and prove a more general result from which the above will follow. The Main Theorem in its general form has somewhat more technical hypotheses which we explain in the next section. In particular the hypothesis of bounded singular orbits is much stronger than what is needed, but easier to state. A neither optimal alternative is to require that all periodic rays land (see Section 2.2).

Along the way we also prove a criterion to establish when the critical point in question is recurrent (see Proposition 1).

\subsection{Statement of results}

To state the general form of our results we first introduce some terminology. In what follows, $f$ is an entire transcendental map and $\Delta$ a bounded Siegel disk of $f$. Let $\bar{\Delta}$ denote the closure of $\Delta$ and $\widehat{\Delta}$ be the complement of the unique unbounded connected component of $\mathbb{C} \backslash \bar{\Delta}$. Equivalently, $\widehat{\Delta}$ is the union of $\Delta, \partial \Delta$ and the bounded components of $\mathbb{C} \backslash \bar{\Delta}$. Observe that $\widehat{\Delta}$ is full by definition. If the boundary of $\Delta$ is a Jordan curve, then $\widehat{\Delta}=\bar{\Delta}$.

Because $\Delta$ is forward invariant, $\partial \Delta$ also is. The bounded components of $\mathbb{C} \backslash \bar{\Delta}$ are called shielded components and are denoted by $H_{i}$. shielded components (see $[\mathbf{H e 2}]$ ) are Fatou components, because $\partial H_{i} \subset \partial \Delta$ and $f(\partial \Delta)=\partial \Delta$; so by the maximum principle the family of iterates on $H_{i}$ is bounded hence normal. For the same reason the images of shielded components are contained in $\widehat{\Delta}$, so $\widehat{\Delta}$ is forward invariant. As a consequence, it is easy to check that $\partial \widehat{\Delta}=\partial \Delta_{\infty}$ where $\Delta_{\infty}$ denotes the unbounded component of $\mathbb{C} \backslash \widehat{\Delta}$. This striking fact does not imply a priori that there are no shielded components, as shown in the lakes of Wada constructions (see $[\mathbf{Y} \mathbf{n}]$ ). Examples of Siegel disks with shielded components have never been found; however, their existence has not been discarded either.

Remark 1.1. The reader may wonder how shielded components may actually look like, or how the preimages of $\Delta$ are related to the presence of critical points in $\partial \Delta$ or outside $\partial \Delta$. Excellent images can be found in $[\mathbf{C R}]$.

In general we allow $f$ to have infinitely many singular values, although in many cases only a finite subset is allowed to interact with $\Delta$.

Definition. (Interacting singular values) We say that only a finite set of singular values $S_{\Delta} \subset S(f)$ is interacting with $\Delta$ if the following conditions are satisfied:

(a) $\omega(s) \cap \widehat{\Delta} \neq \emptyset$ for all $s \in S_{\Delta}$;

(b) $\overline{\bigcup_{s \in S(f) \backslash S_{\Delta}} \omega(s)} \cap \widehat{\Delta}=\emptyset$, and

(c) the singular values in $S(f) \backslash S_{\Delta}$ do not accumulate on $\partial \Delta$.

If only a finite set $S_{\Delta}$ of singular values is interacting with $\Delta$, then the singular values in $S(f) \backslash S_{\Delta}$ are called non-interacting. Escaping singular values, as well as singular values which belong to preperiodic Fatou components whose closure is disjoint from $\partial \Delta$, are non-interacting (if they are in wandering domains, they could). Also note that if $S(f)$ is finite then it is (trivially) true that there are only finitely many singular values interacting with $\Delta$. 
A property that plays a role in a certain part of the proof is the following.

Definition (Separation Property). We say that a holomorphic function $f$ has the Separation Property if the closures of any two periodic components of $F(f)$ may intersect in at most one point.

The Separation Property is satisfied by all polynomials with a connected Julia set $[\mathbf{G M}]$. Entire maps for which an analogue of external rays can be defined and such that all periodic rays land, also have this property $[\mathbf{B F}]$ (see Section $2 \cdot 2$ for details). In particular, functions of finite order in class $\mathcal{B}$ (or compositions thereof) possess dynamic rays in their escaping set [R3S] (see again Section $2 \cdot 2$ for the definition of dynamic rays). It is expected that, unless a periodic ray contains a singular value, it does land, in which case it necessarily has to land at a repelling or parabolic periodic point by the Snail Lemma. This is the case, for example, in polynomial dynamics ([Mi], Theorem 18.10), and for the exponential family [Re3]. In the general case, however, the simplest (and strongest) criterion for all periodic rays to land is to assume that the union of all singular orbits or postsingular set is bounded.

Our main theorem in the most general form reads as follows.

Main Theorem (General form). Let $f$ be an entire function with no wandering domains with bounded orbits and satisfying the Separation Property. Suppose $f$ has a bounded invariant Siegel disk $\Delta$ with rotation number $\theta \in \mathcal{H}$, and such that at most two singular values are interacting with $\Delta$. Then there is a critical point on $\partial \Delta$.

Remark. The hypothesis of no wandering domains can be replaced by the stronger requirement for $f$ to have finitely many singular values since, as we mentioned above, such functions are known not to have wandering domains [EL, GK].

An immediate corollary is the following:

Corollary. Let $f$ be an entire function with no wandering domains and satisfying the Separation Property. If $f$ has no critical values then all Siegel disks with rotation number in $\mathcal{H}$ are unbounded.

In view of the results in $[\mathbf{B F}, \mathbf{R e 1}]$ mentioned above, we recover Herman's Theorem (c) which says that all Siegel disks of the exponential map with rotation number in $\mathcal{H}$ are unbounded.

The proof of the Main Theorem is subdivided into three cases, each of them requiring different hypotheses: In case A we assume that all singular values interacting with $\Delta$ are outside $\widehat{\Delta}$, in case $\mathrm{B}$ we assume that they are all contained in $\widehat{\Delta}$, and in case $\mathrm{C}$ we assume that there is exactly one interacting singular value inside and one outside of $\widehat{\Delta}$. This is the same proof structure that was used in the polynomial case in $[\mathbf{C R}]$. Our contribution in this paper consists of the proof of case B without using Mañe's theorem (see below) and the proof of case $\mathrm{C}$ when there are asymptotic values interacting with the Siegel disk. To make the paper reasonably self-contained, we include the main ideas of the full proof. We remark that the Separation Property and the restriction to two singular values is only necessary for case $\mathrm{C}$.

We now list the three independent statements here named as Propositions A, B and C.

Proposition A (All singular values outside). Let $f$ be an entire map with a bounded Siegel disk $\Delta$ of rotation number $\theta$, and suppose $S(f) \cap \widehat{\Delta}=\emptyset$. Then

(a) $\theta \notin \mathcal{H}$ (Herman);

(b) If furthermore $f$ satisfies the Separation Property and has no wandering domains with bounded orbits, then $\Delta$ has no shielded components.

The first statement is due to Herman as a consequence of his theorem stated above (part (a)). The second statement is actually an addendum since it does not contribute to the proof of the Main Theorem. It is a transcendental counterpart of [Ro, Theorem 3.1] and has a similar proof, using the 
results in $[\mathbf{B F}]$ to ensure that the set of transcendental functions satisfying the Separation Property is non-empty. What the proof of Proposition A shows in the transcendental case is actually that any shielded component must be a wandering domain with bounded orbit, so that if $f$ has no such domains as in case (b), the Siegel disk cannot have shielded components. Wandering domains with bounded orbit are not known to exist, but are not discarded either. It is plausible that they could be excluded under the assumption of having only finitely many singular values interacting with $\Delta$.

We remark that the nonexistence of shielded components does not imply that $\partial \Delta$ is a Jordan curve, as shown by the Siegel disk examples of Chéritat in $[\mathbf{C h}]$, whose boundary is a pseudocircle (thus separating the plane into two components but not being locally connected at any point). These examples however, do not come a priori from a globally defined map.

The case in which all singular values are contained in $\widehat{\Delta}$ is treated in Proposition B.

Proposition B (All singular values inside). Let $f$ be an entire map with a bounded invariant Siegel disk $\Delta$. Suppose that there are only finitely many singular values interacting with $\Delta$, all of which are contained in $\widehat{\Delta}$, and that there are no wandering domains in $\widehat{\Delta}$. Then there is a recurrent critical point on $\partial \Delta$.

In $[\mathbf{C R}]$, the analogous case for polynomials is proved using Mañe's Theorem [Ma3] which ensures the existence of a recurrent critical point whose orbit accumulates on $\partial \Delta$. The transcendental version of this nontrivial result is not yet proven in full generality (see $[\mathbf{R v S}]$ for a certain class of entire maps). Instead, our proof of Proposition B relies on a variation of Fatou's Theorem (see Lemma 3.1), which asserts that all points in the boundary of a Siegel disk must be accumulated by the union of the singular orbits. In the process, we extract the following corollary which we believe might be useful in itself.

Proposition 1 (Recurrent critical points). Let $f$ be an entire function with a bounded invariant Siegel disk $\Delta$. Suppose that there are only finitely many singular values interacting with $\Delta$, all of which are contained in $\partial \Delta$. Then there is at least one recurrent critical point on $\partial \Delta$.

The final case in which there is exactly one interacting singular value in $\widehat{\Delta}$ and one outside $\widehat{\Delta}$ completes the proof of the Main Theorem for functions with only two singular values interacting with $\Delta$.

Proposition C (One inside one outside). Let $f$ be an entire transcendental map with no wandering domains with bounded orbits and which has a bounded invariant Siegel disk $\Delta$ with rotation number $\theta \in \mathcal{H}$. Suppose that $f$ has only two singular values $v, v^{\prime}$ interacting with $\Delta$, and satisfies the Separation Property. If there is exactly one singular value $v$ in $\widehat{\Delta}$, then $v \in \partial \Delta$ and there is a critical preimage $c$ of $v$ on $\partial \Delta$.

The hypothesis of the Main Theorem in its simplified version imply the hypothesis of the Main Theorem in the general form, so that it is enough to prove the latter.

Derivation of the simplified version from the general form. Since $f$ has finitely many singular values, it has no wandering domains $([\mathbf{E L}],[\mathbf{G K}])$. Since it has finite order and bounded set of singular values, it admits dynamic rays ([R3S $]$ ), and its periodic rays land because the singular orbits are bounded ([Re4], $[\mathbf{D e}])$. So by $[\mathbf{B F}], f$ has the separation property (see Section $2 \cdot 2$ ) and $f$ satisfies all the requirement of the main theorem in its general form.

\section{Preliminaries and tools}

\subsection{Mapping properties of entire functions}

Recall from the introduction that a singular value is a point for which there is no neighborhood on 
which all branches of the inverse function are well defined and univalent. In this sense, such a value represents a singularity for $f^{-1}$, although the latter is globally defined only as a multivalued function. A singular value which is not accumulated by other singular values is called an isolated singular value, and points in $\mathbb{C} \backslash S(f)$ are called regular points. Singular values can also have noncritical preimages: if $a$ is a preimage of $s$ such that $f$ is univalent in a neighborhood of $a$, we say that $a$ is a regular preimage of $s$; otherwise, we say that it is a critical preimage.

For the following classification of singularities of $f^{-1}$ see $[\mathbf{B E}]$ and $[\mathbf{I v}]$.

Proposition 2.1 (Classification of singularities). Let $f$ be an entire function, $z \in \mathbb{C}, D(z, r)$ be the disk of radius $r$ centered at $z$. For any $r$ let $U_{r}$ be a component of $f^{-1}(D(z, r))$, chosen such that $U_{r} \subset U_{r^{\prime}}$ if $r<r^{\prime}$. Then only two cases are possible:

(a) $\bigcap_{r} U_{r}=\{p\}, p \in \mathbb{C}$

(b) $\bigcap_{r} U_{r}=\emptyset$

In case $(a), f(p)=z$, and either $f^{\prime}(p) \neq 0$ and $p$ is a regular preimage of $z$, or $f^{\prime}(p)=0$ and $z$ is a critical value. In case $(b)$, the chosen branch $U_{r}$ defines a transcendental singularity over $z, z$ is an asymptotic value for $f$, and we say that $z$ has a preimage at infinity along that given branch.

Singular values can be both critical values or asymptotic values (depending on the selected branch of the inverse). A singular value can be an accumulation point of asymptotic or critical values without being asymptotic or critical itself: in this case, it is a singular value in the sense that not all branches of the inverse are well defined and univalent in any neighborhood, but all its preimages could be regular in the sense of Proposition 2.1. An example of such a point is the value 1 for the function $f(z)=\frac{(z+c) \sin (z)}{z}$ for $c \neq 0$. Indeed, one can check that $f^{\prime}$ does not tend to zero on any curve tending to infinity, so $f$ has no asymptotic values. For large values of $z, f(z)$ is close to $\sin (z)$ and has critical points very close to $(2 k+1) \pi / 2$, with critical values which tend to 1 as $k \rightarrow \pm \infty$.

The next lemma is a basic fact in algebraic topology, see for example $[\mathbf{H a}]$ for general theory about coverings.

Lemma $2 \cdot 2$ (Coverings of $\mathbb{D}$ and $\left.\mathbb{D}^{*}\right)$. Let $U \subset \widehat{\mathbb{C}}, \mathbb{D}$ be the unit disk and $\mathbb{D}^{*}=\mathbb{D} \backslash\{0\}$.

(a) If $f$ is a holomorphic covering from $U \rightarrow \mathbb{D}$, then $U$ is simply connected and $f$ is univalent.

(b) If $f$ is a holomorphic covering from $U \rightarrow \mathbb{D}^{*}$, then either $U$ is biholomorphic to $\mathbb{D}^{*}$ and $f$ is equivalent to $z^{d}$, or $U$ is simply connected and $f$ is the universal covering, hence equivalent to the exponential map.

Sketch of proof. As $f$ is a covering, the fundamental group of $U$ is a subgroup of the fundamental group of $f(U)$ which is either $\mathbb{D}$ or $\mathbb{D}^{*}$.

(a) The fundamental group of $f(U)=\mathbb{D}$ is trivial, hence $U$ is simply connected as well and $f$ is a homeomorphism.

(b) The fundamental group of $U$ is a subgroup of the fundamental group of $\mathbb{D}^{*}$ which is $\mathbb{Z}$, hence it is either trivial or isomorphic to $\mathbb{Z}$. In the first case $f$ is the universal covering and $f$ is equivalent to the exponential map. In the second case, $U$ is the quotient of the upper half plane by a translation, hence $U \sim \mathbb{D}^{*}$ and $f$ is equivalent to $z^{d}$.

Corollary 2.3 (Separating branches). If $f$ is a holomorphic map and $z \in \mathbb{C}$ is an isolated singular value for $f$, then there is a neighborhood $V$ of $z$ such that each component of $f^{-1}(V)$ is simply connected, and is either unbounded or contains at most one critical point. 
Proof. Let $V$ be any simply connected neighborhood of $z$ such that $V \cap S(f)=\{z\}$ which exists because $z$ is not accumulated by singular values. Let $U$ be a component of $f^{-1}(V)$. By Lemma $2 \cdot 2$, either $f: U \rightarrow V$ is univalent, or $f: U \backslash\left\{f^{-1}(z)\right\} \rightarrow V \backslash\{z\}$ is a covering of $\mathbb{D}^{*}$. In this case, either $U$ contains exactly one critical point and $f$ is equivalent to $z^{d}$, or $U$ is unbounded and simply connected and $f$ is equivalent to the exponential.

\subsection{The Separation Property}

In this section we want to give some details about the Separation Property and the class of functions which satisfy it.

Let $\mathcal{B}$ be the class of entire functions with bounded set of singular values, as defined in the introduction. We set

$$
\widetilde{\mathcal{B}}=\left\{f=g_{1} \circ \cdots \circ g_{k} \mid g_{i} \in \mathcal{B} \text { and has finite order }\right\} .
$$

For this class of functions, it is shown in [R3S] that points whose orbits escape to infinity are organized in unbounded curves mapped to each other according to some symbolic dynamics. More precisely, there are maximal injective curves $g_{\mathbf{s}}:(0, \infty) \rightarrow \mathbb{C}$, where $\mathbf{s} \in \mathbb{Z}^{\mathbb{N}}$ is a labelling, such that:

- $\lim _{t \rightarrow \infty}\left|f^{n}\left(g_{\mathbf{s}}(t)\right)\right|=\infty \quad \forall n \geq 0$

- $\lim _{n \rightarrow \infty}\left|f^{n}\left(g_{\mathbf{s}}(t)\right)\right|=\infty$ uniformly in $[t, \infty)$.

These curves are called dynamic rays, or just rays, and are in many ways analogous to external rays of polynomials. If $\lim _{t \rightarrow 0} g_{\mathbf{s}}(t)$ exists, it is said that the ray $g_{\mathbf{s}} l a n d s$. A ray $g_{\mathbf{s}}$ is called periodic if for some $k>0, f^{k}\left(g_{\mathbf{s}}\right) \subset g_{\mathbf{s}}$.

Although it is plausible to think that periodic rays land unless they contain a singular value, for the time being this is only proven for polynomial or exponential dynamics. A sufficient condition for a periodic ray $g_{\mathbf{s}}$ to land (see $[\mathbf{F a}, \mathbf{R e} 4]$ ) is that $g_{s} \cap P(f)=\emptyset$, where $P(f)$ denotes the postsingular set

$$
P(f):=\overline{\bigcup_{s \in S(f)} \bigcup_{n \geq 0} f^{n}(s)}
$$

Hence to ensure that all periodic rays land we may require, for example, that the postsingular set is bounded (see e.g. $[\mathbf{D e}]$ ), although this is a very strong condition.

Under the assumption that $f \in \widetilde{\mathcal{B}}$ and that periodic rays land, it is shown in [BF, Corollary D] that $f$ satisfies the Separation Property. More precisely the following result is proven.

Theorem 2.4 (Separation Theorem). If $f \in \widetilde{\mathcal{B}}$ and periodic rays land, any two periodic Fatou components can be separated by two periodic rays landing at the same point. Hence the boundaries of any two bounded periodic Fatou components intersect in at most one point.

A consequence of Theorem 2.4 is the following corollary [BF, Corollary E], which is directly related with our problem at hand.

Corollary 2.5 (Preperiodic shielded components). If $f$ is an entire function satisfying the Separation Property then any shielded component of a bounded forward invariant Siegel disk is preperiodic to the Siegel disk itself or a wandering domain with bounded orbit.

The corollary follows quite directly from the Separation Theorem. If a shielded component $H$ is periodic, its boundary can intersect $\partial \Delta$ in at most one point. But $\partial H \subset \partial \Delta$ and hence this is impossible. 
2.3. Properties of hats

Given a bounded set $X \subset \mathbb{C}$, recall that $\widehat{X}$ denotes the union of $\bar{X}$ and the bounded components of $\mathbb{C} \backslash \bar{X}$. (By the name of component we always mean connected component.) Observe that $\bar{X}$ is always closed and full.

The following proposition summarizes the facts that we shall use about "hats". They will only be used in the proof of Proposition C, in Section 4. Most of them can be found or deduced from results in [CR, Section 2], but we include the proof here for completeness and self-containment.

Proposition 2.6. Let $X, Y$ be bounded subsets of $\mathbb{C}, V$ be a simply connected neighborhood of $\bar{X}$. Then the following facts hold:

(1) $Y \subseteq \widehat{X} \Longleftrightarrow \bar{Y} \subseteq \widehat{X} \Longleftrightarrow \widehat{Y} \subseteq \widehat{X}$.

(2) Let $g$ be a univalent function defined in $V$. Then $\widehat{g(X)}=g(\widehat{X})$.

(3) Let $f$ be an entire function without singular values in $V \backslash \widehat{X}$. Let $U$ be a bounded connected component of $f^{-1}(V)$. For a set $A$ compactly contained in $V$ we set $f_{U}^{-1}(A)=f^{-1}(A) \cap U$. Then

$$
\widehat{f_{U}^{-1}(X)}=f_{U}^{-1}(\widehat{X})
$$

Proof.

(1) This follows because $\widehat{X}$ is both closed and full.

(2) Observe that

$$
\begin{aligned}
\widehat{g(X)} & =\overline{g(X)} \cup\{\text { bounded components of } \mathbb{C} \backslash \overline{g(X)}\} \\
\widehat{X} & =\bar{X} \cup\{\text { bounded components of } \mathbb{C} \backslash \bar{X}\} .
\end{aligned}
$$

We first show that $\widehat{g(X)} \subseteq g(\widehat{X})$. If $z \in \overline{g(X)}, g^{-1}(z) \in \bar{X}$ because $g$ is a homeomorphism, hence $g^{-1}(z) \in \widehat{X}$ and $z \in g(\widehat{X})$. If $z$ is in a bounded component of $\mathbb{C} \backslash \overline{g(X)}$ then $g^{-1}(z)$ belongs to a bounded component of $\mathbb{C} \backslash \bar{X}$ by the maximum principle hence $g^{-1}(z) \in \widehat{X}$ and $x \in g(\widehat{X})$.

We now show that $\widehat{g(X)} \supseteq g(\widehat{X})$. If $z \in g(\widehat{X})$ then $g^{-1}(z) \in \widehat{X}$. If $g^{-1}(z) \in \bar{X}$ then $z \in g(\bar{X})=\overline{g(X)}$ because $g$ is a homeomorphism, hence $z \in \widehat{g(X)}$. Otherwise $g^{-1}(z)$ is in a bounded component of $\mathbb{C} \backslash \bar{X}$ hence $z$ is in a bounded component of $\mathbb{C} \backslash \overline{g(X)}$ and $z \in \widehat{g(X)}$.

(3) Let $\left\{X_{i}\right\}_{i}$ be the components of $f^{-1}(X)$ which are contained in $U$, and $X^{\prime}=\bigcup_{i} X_{i}=f_{U}^{-1}(X)$. By definition $f\left(X^{\prime}\right)=X$. Since $f_{U}^{-1}(X)$ has a finite number of components, $\partial X^{\prime}=\bigcup_{i} \partial X_{i}$. Since $f: U \rightarrow V$ is open and proper it follows that $\overline{X^{\prime}} \subset U, f\left(\overline{X^{\prime}}\right)=\bar{X}$ and $f\left(\partial X^{\prime}\right)=\partial X$. Consequently, since $f$ has no poles, by the maximum principle $f$ maps bounded components of $\mathbb{C} \backslash \overline{X^{\prime}}$ to bounded components of $\mathbb{C} \backslash \bar{X}$. Conversely, if $W$ is a bounded component of $\mathbb{C} \backslash \bar{X}$, then $f_{U}^{-1}(W)$ consists of a finite number of bounded components of $\mathbb{C} \backslash \overline{X^{\prime}}$.

We first show that $\widehat{X^{\prime}} \subseteq f_{U}^{-1}(\widehat{X})$. If $z \in \overline{X^{\prime}}, f(z) \in \bar{X}$ hence $f(z) \in \widehat{X}$ and $z \in f_{U}^{-1}(\widehat{X})$ by definition. If $z$ is in a bounded component of $\mathbb{C} \backslash \overline{X^{\prime}}$ then $f(z)$ belongs to a bounded component of $\mathbb{C} \backslash \bar{X}$ hence $f(z) \in \widehat{X}$. Then by definition $z \in f_{U}^{-1}(\widehat{X})$.

We now show that $\widehat{X^{\prime}} \supseteq f_{U}^{-1}(\widehat{X})$. If $z \in f_{U}^{-1}(\widehat{X})$ then $f(z) \in \widehat{X}$. If $f(z) \in \bar{X}$, then $z \in \overline{X^{\prime}} \subset \widehat{X^{\prime}}$. Otherwise $f(z)$ is in a bounded component of $\mathbb{C} \backslash \bar{X}$, hence $z$ is in a bounded component of $\mathbb{C} \backslash \overline{X^{\prime}}$. 


\section{Proof of Propositions A, B and Proposition 1}

Let $\Delta$ be a bounded forward invariant Siegel disk of $f$, and $H_{i}$ the shielded components, that is the bounded components of $\mathbb{C} \backslash \bar{\Delta}$. By definition,

$$
\widehat{\Delta}=\partial \Delta \cup \Delta \cup \bigcup_{i} H_{i}
$$

Let $\widetilde{\Delta}$ be the component of $f^{-1}(\widehat{\Delta})$ containing $\widehat{\Delta}$.

The proof of part (a) in Proposition A is due to Herman ([He2]); we sketch it for completeness and refer to $[\mathbf{C R}$, Section 5] for details. We give a proof of part (b).

We call a compact set $K$ locally backward invariant if there is a neighborhood $V$ of $K$ and a connected component $U$ of $f^{-1}(V)$ such that $U \cap f^{-1}(K)=K$.

Proof of Proposition A. Since $\widehat{\Delta}$ is full and contains no singular values, and $S(f)$ is a closed set, there exists a simply connected neighborhood $V$ of $\widehat{\Delta}$ such that $\bar{V} \cap S(f)=\emptyset$. Let $U$ be the component of $f^{-1}(V)$ containing $\widetilde{\Delta}$.

(a) By Lemma 2.2, $f: U \rightarrow V$ is a homeomorphism, hence $\widetilde{\Delta}=\widehat{\Delta}$. The complement of $\widehat{\Delta}$ can be uniformized to the complement of the unit disk $\overline{\mathbb{D}}$, and the uniformization map conjugates $f$ to an analytic map $g$ defined in a small annulus around $\overline{\mathbb{D}}$. Because $\widehat{\Delta}$ is locally backward invariant, using Schwarz's reflection principle $g$ can be extended to an analytic map in a neighborhood of $\mathbb{S}^{1}$, and its restriction to $\mathbb{S}^{1}$ is an analytic circle map, which has degree one because $f$ is univalent in a neighborhood of $\widehat{\Delta}$. The rotation number of $g$ can be shown to be the same as the rotation number of $\Delta$, i.e. $\theta$; if $\theta \in \mathcal{H}$, by Herman's theorem [He1] the map $g$ can be linearized via an analytic map. Then the Riemann map transports it to a linearization for $f$ in a neighborhood of the Siegel disk, contradicting its maximality.

(b) As in part (a), by Lemma $2 \cdot 2$ the map $f$ from $U \rightarrow V$ is univalent, hence the unique preimage of $\Delta$ in $U$ is $\Delta$ itself. Using Corollary 2.5 and the nonexistence of wandering domains, any shielded component of $\widehat{\Delta}$ is preperiodic to $\Delta$. So if a shielded component $H$ exists, there exists $n>0$ such that $f^{n}(H)=\Delta$ and $f^{n-1}(H) \neq \Delta$. Hence $f^{n-1}(H)$ is a preimage of $\Delta$ in $U$, which is a contradiction.

We now proceed to prove Propositions B and Proposition 1.

Proposition B relies strongly on the following lemma $3 \cdot 1$, which is a modification of Fatou's Theorem as quoted in the introduction. The proof is the same for rational and for transcendental functions.

Lemma 3.1. Let $f$ be an entire function with a bounded invariant Siegel disk $\Delta$. Assume that there are only finitely many singular values interacting with $\Delta$. Suppose that there exists a point $z \in \partial \Delta$ and a neighborhood $V$ of $z$ such that $\omega(s) \cap V=\emptyset$ for all singular values which are in not in $\partial \Delta$. Then there is at least one recurrent critical point on $\partial \Delta$.

Remark. Under weaker hypotheses, it follows from [RvS, Corollary 2.9] that the the boundary of $\Delta$ is contained in the $\omega$-limit set of a recurrent or almost recurrent (see $[\mathbf{R v S}]$ for a definition) singular value, provided that the function in question has finitely many singular values. But nothing is said (or easily deduced) about critical points.

Proof. Let $z, V$ be as in the statement. By Fatou's theorem, and since there are finitely many singular values interacting with $\Delta$, there exists at least one singular value $v_{1} \in \partial \Delta$ whose orbit accumulates on 
$z$, and there are only finitely many singular values in $\partial \Delta$. So, let $v_{1} \ldots v_{q} \subset \partial \Delta$ be the finitely many singular values whose forward orbits intersect $V$. By hypothesis, up to restricting $V$, it contains no iterates of any singular value other than those of $v_{1}, \ldots, v_{q}$. Up to taking a smaller $V$ we can assume that it contains at most one singular value. Let $\varepsilon<\min _{i \neq j \leq q}\left|v_{i}-v_{j}\right|$, and such that there are no other singular values in an $\varepsilon$-neighborhood of $\partial \Delta$.

We first show that there is at least one critical point in $\partial \Delta$. Suppose by contradiction that there are no critical points in $\partial \Delta$. Then all preimages of each $v_{i}$ which are contained in $\partial \Delta$ are regular, and since $\partial \Delta$ is bounded and $f: \partial \Delta \rightarrow \partial \Delta$ is surjective, each $v_{i}$ has at least one preimage in $\partial \Delta$. Let $D_{i}$ be the Euclidean disk of radius $\varepsilon$ centered at $v_{i}$, and let $\psi_{i}$ be the unique branch of $f^{-1}$ defined on $D_{i}$ which maps $D_{i} \cap \Delta$ to $\Delta$ (see the proof of Corollary 14.4 in [Mi]). Since each singular value has only regular preimages, $\psi_{i}$ is univalent, and for each $i$ there exists $a_{i} \in \partial \Delta$ which is the unique regular preimage of $v_{i}$ such that $\psi_{i}\left(v_{i}\right)=a_{i}$.

Claim. For each $n \in \mathbb{N}$ there is a unique univalent branch $\phi_{n}$ of $f^{-n}$ defined on $V$ which maps $V \cap \Delta$ to $\Delta$.

See also the proof of [Mi, Corollary 14.4]. For $n=1$ the claim is true: If $V$ contains no singular values, since it is simply connected all its preimages are univalent and at least one of them maps $V \cap \Delta$ to $\Delta$. If there is a singular value in $V$, say $v_{1}$, it is unique by assumption, and the branch $\psi_{1}$ extends to $V$ so that we can define $\phi_{1}$ to be this extension.

Now assume that for some $n$ the branch $\phi_{n}$ is well defined, univalent and maps $V \cap \Delta$ to $\Delta$ and consider $\phi_{n}(V)$, which is therefore simply connected. If there are no singular values in $\phi_{n}(V)$ the claim is evidently true also for $n+1$. Since $f^{n}(s) \cap V=\emptyset$ for all $s \in S(f)$ which is different from $v_{1}, \ldots, v_{q}$, the only singular values that might belong to $\phi_{n}(V)$ are $v_{1}, \ldots, v_{q}$. Let us suppose that $v_{1}, \ldots, v_{k} \in \phi_{n}(V)$. Then, for each $1 \leq i \leq k$ consider the inverse branch $\psi_{i}$ defined in $D_{i}$ which maps $\psi_{i}\left(v_{i}\right)=a_{i}$. Each branch $\psi_{i}$ can be extended univalently to any simply connected open subset of $\phi_{n}(V)$ not containing any other singular value.

Since $a_{i} \in \partial \Delta$ there is a sequence of points $d_{n} \in \Delta$ converging to $a_{i}$ such that $f\left(d_{n}\right) \rightarrow v_{i}$, so by injectivity of $f$ on $\Delta$ and by the identity principle each extended $\psi_{i}$ is the unique inverse branch mapping $\Delta$ to $\Delta$ in its domain of definition. Since any two such branches $\psi_{i}, \psi_{j}$ overlap on some open set, by the identity principle they coincide and patch to a univalent inverse branch $\psi$ defined on all of $\phi_{n}(V)$. It is now clear that $\psi$ is the unique branch of the inverse mapping $\phi_{n}(V) \cap \Delta$ to $\Delta$, so that $\phi_{n+1}$ is well defined on $V$ and maps $V \cap \Delta$ to $\Delta$. This concludes the proof of the Claim.

The family $\left\{\phi_{n}\right\}$ is normal because it omits three values. Moreover it does not converge to infinity, so up to passing to a subsequence we can assume that it converges to a univalent function $\phi$. As $\phi_{n}(V \cap \Delta) \subset \Delta$ for all $n$, it follows that $\phi(V \cap \Delta) \subset \Delta$ and $\phi$ is non-constant (because $\phi(V) \cap \mathcal{J}(f) \neq \emptyset$, and grand orbits of points in $\Delta$ do not accumulate on the Julia set), hence open. Hence there is a point $y \in \partial \Delta \cap \phi(V)$ such that a neighborhood of $y$ is mapped into $V$ under infinitely many iterates, contradicting $y \in \mathcal{J}(f)$. We therefore conclude that there exists at least one singular value, say $v_{1}$, whose preimage in $\partial \Delta$ is non-regular. If $v_{1}$ is an asymptotic value, then its preimage is at infinity and hence $\Delta$ is unbounded. It follows that $v_{1}$ is a critical value and its preimage in $\partial \Delta$ is a critical point, as we wanted to show. By construction, the orbit of $v_{1}$ accumulates on $z$.

We now show recurrence. Suppose that there are $q$ critical points $\left\{c_{i}\right\}$ on $\partial \Delta$ whose orbits accumulate on $z$, and let $\mathcal{C}$ be the collection of these critical points, which is non-empty by the previous part of the proof. None of them is contained in the accumulation set of the orbit of any singular value $v \notin \partial \Delta$, for otherwise the orbit of $v$ would also accumulate on $z$ contradicting the assumption on $z$. So each 
$c_{i} \in \mathcal{C}$ satisfies the hypotheses of this lemma hence it is in the accumulation set of the orbit of some (possibly different) critical point $c_{j} \in \partial \Delta$; but since the orbit of $c_{j}$ accumulates on $c_{i}$ whose orbit accumulates on $z$, then the orbit of $c_{j}$ accumulates on $z$ as well and hence $c_{j} \in \mathcal{C}$. Let $c_{1}$ be any of the critical points in $\mathcal{C}, c_{2}$ be a critical point whose orbit accumulates on $c_{1}, c_{3}$ be a critical point whose orbit accumulates on $c_{2}$ and so on. Since $\mathcal{C}$ is finite, $c_{q}=c_{n}$ for some $n<q$. But then iterates of $c_{q}=c_{n}$ accumulate on $c_{q-1}$ whose iterates accumulate on $c_{q-2}$ and so on until $c_{n}$, hence by extracting a diagonal sequence of iterates, iterates of $c_{n}$ accumulate on $c_{n}$ itself giving recurrence. This concludes the proof of Lemma 3.1 .

Proposition 1 in the introduction follows almost directly from Lemma $3 \cdot 1$. It is a weaker statement but somewhat easier to state. We are now ready to prove Proposition B.

Proof of Proposition B. Let $S_{\Delta}$ be the finite set of singular values interacting with $\Delta$. For any singular value $s \notin S_{\Delta}$ we have that $\omega(s) \cap \partial \Delta=\emptyset$, while for any $s \in S_{\Delta} \cap F(f)$, by the classification of Fatou components and absence of wandering domains in $\widehat{\Delta}$, either $\omega(s) \cap \partial \Delta$ is empty or it consists of a finite parabolic orbit. So there is a point $z \in \partial \Delta$ which is not contained in $\omega(s)$ for any $s \notin \partial \Delta$ hence satisfying the hypothesis of Lemma $3 \cdot 1$. By this lemma, there is a recurrent critical point on $\partial \Delta$.

\section{Proof of Proposition $C$}

We now assume that $f$ has only two singular values $v, v^{\prime}$ interacting with $\Delta$, where $\Delta$ is a bounded forward invariant Siegel disk for $f$ with rotation number $\theta \in \mathcal{H}$. We also assume that periodic Fatou components can be separated and that $f$ has no wandering domains in $\widehat{\Delta}$. In this Section we prove that if there is exactly one singular value $v$ in $\widehat{\Delta}$, then there is at least one critical preimage $c$ of $v$ on $\partial \Delta$. Recall that $\widetilde{\Delta}$ denotes the component of $f^{-1}(\widehat{\Delta})$ containing $\widehat{\Delta}$.

If $v$ is a critical value, our setup is equivalent to the one for polynomials and the proof is analogous. We reproduce it here for completeness, referring to $[\mathbf{C R}]$ only for a part of the argument which is quite technical and goes through directly in our case. The new part is the proof in the case that $v$ is an asymptotic value.

The proof of Proposition C uses the following two results.

Lemma $4 \cdot 1$ (Regular preimages). Let $f$ be an entire function, $\Delta$ a bounded Siegel disk for $f$. Suppose that $S(f) \cap \widehat{\Delta}=\{v\}$ where $v$ is an isolated singular value with a regular preimage in $\widetilde{\Delta}$. Then $\widetilde{\Delta}=\widehat{\Delta}$ and $\theta \notin \mathcal{H}$.

Proof. To show the first claim observe that since $\widehat{\Delta}$ is full and $v$ is an isolated singular value, there is a simply connected neighborhood $V$ of $\widehat{\Delta}$ such that $\bar{V} \cap S(f)=\{v\}$; let $U$ be the component of $f^{-1}(V)$ containing $\widetilde{\Delta}$. The map $f: U \backslash f^{-1}(v) \rightarrow V \backslash\{v\}$ is a covering, hence by Lemma $2 \cdot 2, f^{-1}(v)$ is either empty or a single point. By hypothesis it cannot be empty and it is a regular preimage, thus $f: U \rightarrow V$ is a homeomorphism and $\widetilde{\Delta}=\widehat{\Delta}$. The proof of Proposition A can then be repeated to show that $\theta \notin \mathcal{H}$.

The proof of Proposition C uses a theorem of Mañé about hyperbolicity of circle maps ([Ma1, Theorem A],[Ma2]).

Theorem $\mathbf{4} \cdot \mathbf{2}$ (Hyperbolicity of circle maps). Let $g: \mathbb{S}^{1} \rightarrow \mathbb{S}^{1}$ be a $C^{2}$ map. Let $\Lambda \subset \mathbb{S}^{1}$ be a forward invariant compact set which does not contain critical points or non-repelling periodic points; then either $\Lambda=\mathbb{S}^{1}$ and $g$ is topologically equivalent to a rotation, or $\Lambda$ is a hyperbolic set, that is there exist $\bar{k}, \eta>1$ such that for all $k>\bar{k}$ and all $z \in \Lambda,\left|\left(g^{k}\right)^{\prime}(z)\right|>\eta$. 
Proof of Proposition $C$. Let $V \supset \widehat{\Delta}$ be a simply connected open set such that $V \cap S(f)=\{v\}$. Let $U$ be the component of the preimage of $V$ which contains $\widehat{\Delta}$. As there is only one singular value $v \in V$ which has no regular preimage by Lemma $4 \cdot 1$, by Lemma $2 \cdot 2$ either $f: U \rightarrow V$ is equivalent to $z^{d}$ and $v$ has only one preimage in $U$ or $f: U \rightarrow V$ is equivalent to the exponential map, $v$ is an asymptotic value, and $\widetilde{\Delta}$ is unbounded.

Recall that $\widehat{\Delta}$ decomposes like

$$
\widehat{\Delta}=\partial \Delta \cup \Delta \cup \bigcup_{i} H_{i}
$$

and that $f: \partial \Delta \rightarrow \partial \Delta$ as well as $f: \Delta \rightarrow \Delta$ are surjective. Hence $v$ cannot belong to $\Delta$, or it would need to have a regular preimage in $\Delta$, which by Lemma $4 \cdot 1$ would imply that $\theta \notin \mathcal{H}$. If $v \in \partial \Delta$ by surjectivity of $\left.f\right|_{\partial \Delta}$ it has a finite preimage on $\partial \Delta$, hence $f: U \rightarrow V$ cannot be equivalent to the exponential map. Again by Lemma $4 \cdot 1$, the preimage of $v$ in $\partial \Delta$ cannot be regular; so $v$ is a critical value and has a critical preimage on $\partial \Delta$ and there is nothing else to prove.

So the only case left is the one in which $v \in H^{v}$ for some shielded component $H^{v}$. In this case, $v$ could be either critical or asymptotic but, by the discussion above, either $v$ has a unique preimage in $\widetilde{\Delta}$ and this preimage is a critical point or $v$ has no preimage in $\widetilde{\Delta}$. The rest of the proof is devoted to show that the condition $v \in H^{v}$ leads to a contradiction.

Case 1: $f: U \rightarrow V$ is equivalent to the exponential map. This is to say that $v$ is an asymptotic value. Necessarily $\widetilde{\Delta}$ and hence $U$ are unbounded. Call $\Delta_{i}$ the components of $f^{-1}(\Delta)$ which are contained in $U$. The $\Delta_{i}$ 's are all preimages of the same bounded set under a holomorphic map, so they do not accumulate on any compact set. Thus showing that $\Delta_{i} \subset \widehat{\Delta}$ for infinitely many $i \in \mathbb{Z}$ would contradict boundedness of $\widehat{\Delta}$.

Since the map $f: U \rightarrow V \backslash\{v\}$ is equivalent to the exponential map, and the map $z \rightarrow e^{z}$ has a transitive infinite cyclic group of automorphisms which is isomorphic to $\mathbb{Z}$ (generated by translations by $2 \pi i$ ), it follows that $f$ also has a transitive infinite cyclic group of automorphisms $G$ generated by some element $\rho \in G$. Up to labelling, $\Delta_{i}=\rho^{i}(\Delta)$ for each $i \in \mathbb{Z}$. By Corollary $2 \cdot 5, H^{v}$ is preperiodic to $\Delta$ so there is some $k$ such that $f^{k}\left(H^{v}\right)=\Delta$, and $f^{k-1}\left(H^{v}\right)=\Delta_{m}$ for some preimage $\Delta_{m} \neq \Delta$ of the Siegel disk, which is also a shielded component of $\widehat{\Delta}$ because $H_{v} \subset \widehat{\Delta}$ which is forward invariant. By Proposition $2 \cdot 6$ part $(1), \rho^{m}(\Delta)=\Delta_{m} \subset \widehat{\Delta} \Rightarrow \widehat{\rho^{m}(\Delta)} \subset \widehat{\Delta}$, and by Proposition 2.6 part (2) since $\rho^{m}$ is a homeomorphism, $\rho^{m}(\widehat{\Delta})=\widehat{\rho^{m}(\Delta)}$ so $\rho^{m}(\widehat{\Delta}) \subset \widehat{\Delta}$. Now, for any $q \in \mathbb{N}$

$$
\rho^{m q}(\widehat{\Delta})=\rho^{m(q-1)} \rho^{m}(\widehat{\Delta}) \subset \rho^{m(q-1)}(\widehat{\Delta}) \subset \ldots \subset \widehat{\Delta} .
$$

But then, for any $q \in \mathbb{N}$, we have that $\rho^{m q}(\Delta) \subset \rho^{m q}(\widehat{\Delta}) \subset \widehat{\Delta}$. It follows that there are infinitely many $\Delta_{i} \subset \widehat{\Delta}$ contradicting boundedness of $\widehat{\Delta}$.

Case 2: $f: U \rightarrow V$ is equivalent to $z^{d}$.

Then the preimage of $v$ is a finite critical point $c \in \widehat{\Delta}$. Since $f$ is equivalent to $z^{d}$ for some $d \geq 2$ it is a proper map, so $U$ (and hence $\widetilde{\Delta}$ ) is bounded.

Once we observe that the shielded component $H_{v}$ has to be preperiodic to $\Delta$ because there are no wandering domains in $\widehat{\Delta}$, this case is totally analogous to the polynomial case which was proven in $[\mathbf{C R}]$. The transcendentality of $f$ plays no role in this setup which can be solved with a local approach. Nevertheless we include the main ideas here for completeness.

The outline of the proof is the following: we first show that $\widetilde{\Delta}=\widehat{\Delta}$, then uniformize $\mathbb{C} \backslash \widehat{\Delta}$ to find a 
map $g$ which is conjugate to $f$ and which extends to an analytic circle map of degree $d$. We show that the periodic cycles of the induced circle map are all repelling by using the fact that if there existed a nonrepelling one, the remaining singular value would have to both be attracted to the nonrepelling cycle and accumulate on $\partial \Delta$ by Fatou's theorem (see the Introduction), which is a contradiction. We can then use Theorem 4.2 to deduce that $f$ has a polynomial-like restriction in $U$ and conclude the proof using Fatou's theorem.

The first goal is to show that $\widetilde{\Delta}=\widehat{\Delta}$. Since $f$ is equivalent to $z^{d}$ it has a cyclic symmetry group $G$ isomorphic to $\mathbb{Z} / d \mathbb{Z}$ generated by some element $\rho$. Like in Case 1 , let $\Delta_{i}=\rho^{i}(\Delta)$ denote the preimages of $\Delta$ in $U$. By Proposition $2 \cdot 6$ part (3), $\widetilde{\Delta}=\widehat{\widehat{U}_{i}}$. Using Corollary 2.5, like in the case where $v$ is asymptotic, we can find a preimage $\Delta_{m}$ of $\Delta$ which is contained in $\widehat{\Delta}$. If $c$ (the preimage of $v$ in $U$ ) is a simple critical point, this concludes the proof that $\widetilde{\Delta}=\widehat{\Delta}$, because there are exactly two preimages $\Delta$ and $\Delta^{\prime}$ of $\Delta$ and both are contained in $\widehat{\Delta}$. If $c$ has a higher multiplicity, the symmetry arguments are more involved: we refer to [CR, Proposition 35 and Subsection 7.1-7.3] for the proof of the fact that $\widetilde{\Delta}=\widehat{\Delta}$.

Now, as in the proof of Proposition A, the complement of $\widehat{\Delta}$ can be uniformized to $\mathbb{C} \backslash \overline{\mathbb{D}}$ via a holomorphic map $\Phi$, in order to get, by Schwarz reflection, an analytic map $g$ defined in a neighborhood of $\mathbb{S}^{1}$, such that $\left.g\right|_{\mathbb{S}^{1}}$ is an analytic circle map with no critical points (see Lemma 44 and Section 2.3 in $[\mathbf{C R}])$. As $g$ is conjugate to $f$ in a small neighborhood of the unit disk, using the fact that $f: U \rightarrow V$ has degree $d$, it can be shown that also $g$ has degree exactly $d$ on $\mathbb{S}^{1}$ [CR, Lemma 44]. Now suppose that $g$ has a non-repelling periodic point $x$ of period $q$ in $\mathbb{S}^{1}$. Then by the Snail Lemma ([Mi], Lemma 16.2), since the unit circle is invariant $x$ cannot be a Cremer point, so either $x$ is an attracting periodic point or a parabolic point (incidentally, of multiplier equal to 1 under $f^{q}$ ) for the locally defined holomorphic map $g$. If $x$ is attracting, it is contained in its own attracting basin under $g^{q}$, which hence intersects $\mathbb{C} \backslash \overline{\mathbb{D}}$. If $x$ is parabolic, since $g$ commutes with the reflection with respect to the unit circle, there are points in the parabolic basin on both sides of the circle. So in both cases there is an open set $\Omega$ of points in $\mathbb{C} \backslash \overline{\mathbb{D}}$ that tend to $x$ under iteration of $g$, and its pullback $\Phi(\Omega)$ is an open set $B$ of points in $\mathbb{C} \backslash \widehat{\Delta}$ tending to $\partial \Delta$ under iteration of $f$. It follows that $B$ is a subset of a periodic Fatou component for $f$, which is either attracting or parabolic. Then the orbit of that periodic Fatou component has to contain a singular value, which has to be $v^{\prime}$ because $f^{n}(v) \in \Delta$ for $n$ large enough. So the orbit of $v^{\prime}$ under $f$ cannot accumulate on any point on $\partial \Delta$ except at finitely many points. Since only $v, v^{\prime}$ are interacting with $\Delta$, and the orbit of $v$ is eventually contained in $\Delta$ hence cannot accumulate on $\partial \Delta$, this contradicts Fatou's Theorem.

So there are no non-repelling periodic points on $\mathbb{S}^{1}$ and $g$ is hyperbolic on $\mathbb{S}^{1}$. Hence there is a neighborhood $U_{\varepsilon}$ of $\mathbb{S}^{1}$ such that $g\left(U_{\varepsilon}\right) \supset U_{\varepsilon}$. Using $\Phi^{-1}$, the set $U_{\varepsilon} \cap(\mathbb{C} \backslash \overline{\mathbb{D}})$ can be pulled back to the dynamical plane of $f$ to get a neighborhood $U_{f}$ of $\widehat{\Delta}$ which is mapped outside itself under $f$, making the restriction of $f$ to $U_{f}$ a polynomial-like map with a unique critical point $c$. Then by Fatou's theorem, $\partial \Delta \subset \omega(v)$, contradicting the fact that $v \in H^{v}$ hence its orbit is eventually contained in $\Delta$ and cannot accumulate on the boundary.

Acknowledgements. We are very thankful to Arnaud Chéritat and Pascale Roesch for sharing with us the ideas of their work on bicritical polynomials, as well as their manuscript which led to this present work. We also thank Walter Bergweiler and Lasse Rempe Gillen for useful comments about the manuscript. The first author was partially supported by the ERC grant HEVO - Holomorphic Evolution Equations n. 277691 and by the SIR grant NEWHOLITE - New methods in holomorphic 
iteration n. RBSI14CFME. The second author was partially supported by the Catalan grant2014SGR555, and by the Spanish grants MTM2014-52209-22-2-P and MDM-2014-0445 Maria de Maeztu.

\section{REFERENCES}

[BF] A. M. Benini and N. FAGella. A separation theorem for entire transcendental maps. Proc. Lon. Math. Soc. 110 (2015), 291-324.

[Be] W. Bergweiler. Iteration of meromorphic functions. Bull. Amer. Math. Soc. (N.S.) 29 (1993), no. 2 , $151-188$.

[BE] W. Bergweiler and A. Eremenko. On the singularities of the inverse of a meromorphic function of finite order. Rev. Mat. Iberoamericana 11 (1995), no. 2, 355-373.

[Bi] C. J. Bishop. Constructing entire functions by quasiconformal folding. Acta Math. 214 (2015), 1-60.

[Ch] A. ChÉritat. Relatively compact Siegel disks with non-locally connected boundaries. Mathematische Annalen 349 (2011), 529-542.

[CR] A. ChÉritat and P. Roesch. Herman's condition and Siegel disks of bi-critical polynomials. Comm. Math. Phys. 344 (2016), no. 2, 397-426.

[De] A. Deniz. A landing theorem for periodic dynamic rays for transcendental entire maps with bounded post-singular set. J. Difference Equ. Appl. 20 (2014), 1627-1640.

[EL] A. Eremenko and M. Lyubich. Dynamical properties of some classes of entire functions. Ann. Inst. Fourier (Grenoble) 42 (1992), no. 4, 989-1020.

[Fa] N. Fagella. Dynamics of the complex standard family. J. Math. Anal. Appl. 229 (1999), no.1, 1-31.

[Gh] E. GHys. Transformations holomorphes au voisinage d'une courbe de Jordan. C.R. Acad. Sc. Paris 289 (1984), 383-388.

[GK] L. Goldberg and L. Keen. A finiteness theorem for a dynamical class of entire functions. Ergodic Theory Dynam. Systems 6 (1986) no.2, 183-192.

[GM] L. Goldberg and J. Milnor. Fixed points of polynomial maps. Part II. Fixed point portraits. Ann. Sci. École Norm. Sup. (4) 26 (1993), no. 1, 51-98.

[GS] J. Graczyk and G. Światek. Siegel disks with critical points on their boundaries. Duke Math. J. 119 (2003), no.3, 189-196.

[Ha] A. Hatcher. Algebraic topology. Cambridge University Press, Cambridge, 2002.

[He1] M.-R. Herman. Sur la conjugaison différentiable des difféomorphismes du cercle á des rotations. Inst. Hautes Études Sci. Publ. Math. 49, (1979) 5-233.

[He2] M. R. Herman. Are there critical points on the boundaries of singular domains? Comm. Math. Phys. 99 (1985), no. 4, 593-612.

[He3] M. Herman. Conjugaison quasi-symétrique des difféphismes du cercle et applications aux disques singuliers de Siegel, manuscript, (1986).

[He4] M. Herman. Conjugaison quasi-symétrique des homeomorphismes analytiques du cercle à des rotations, manuscript 1987.

[Iv] F. Iversen. Recherches sur les fonctions inverses. Comptes Rendus 143 (1906), 877-879; Math Werke, no. $1,655-656$.

[Ma1] R. MAÑÉ. Hyperbolicity, sinks and measure in one-dimensional dynamics. Comm. Math. Phys. 100 (1985), no. 4 495-524.

[Ma2] R. MAÑé. Erratum: Hyperbolicity, sinks and measure in one-dimensional dynamics. Comm. Math. Phys. 112 ( 1987), no. 4, 721-724.

[Ma3] R. Mañé. On a theorem of Fatou. Bol. Soc. Brasil. Mat. (N.S.) 24 (1993), no. 1, 1-11.

[Mi] J. Milnor. Dynamics in one complex variable. Annals of Mathematics Studies (2006), Princeton University Press.

[Re1] L. Rempe. Dynamics of Exponential Maps, doctoral thesis, Christian-Albrechts-Universität Kiel, (2003) http://http://eldiss.uni-kiel.de/macau/receive/dissertation_diss_00000781.

[Re2] L. Rempe. On a question of Herman, Baker and Rippon concerning Siegel disks. Bull. Lon. Math. Soc. 36 (2004), 516-518.

[Re3] L. Rempe. A landing theorem for periodic rays of exponential maps. Proc. Amer. Math. Soc. 134(9) (2006), 2639-2648.

[Re4] L. Rempe. Siegel disks and periodic rays of entire functions. J. Reine Angew. Math. 624 (2008), 81-102.

[RvS $]$ L. Rempe and S. VAn Strien. Absence of line fields and Mañé's theorem for non-recurrent transcendental functions. Trans. Amer. Math. Soc. 363 (2011), no. 1, 203-228.

[R3S] G. Rottenfusser, J. RüCKert, L. Rempe and D. Schleicher. Dynamic rays of bounded-type entire functions. Annals of Mathematics 173 (2010), 77-125.

[Ro] J. T. Rogers. Diophantine conditions imply critical points on the boundaries of Siegel disks of polynomials. Comm. Math. Phys. 195 (1998), no. 1, 175-193. 
[Yo] J.-C. Yoccoz. Analytic linearization of circle diffeomorphisms. Dynamical systems and small divisors (Cetraro, 1998), volume 1784 of Lecture notes in Math., Springer, Berlin (2002), 125-173.

[Yn] K. Yoneyama. Theory of Continuous Set of Points. Tohoku Math. J. 12 (1917), 43-158. 